\title{
Avaliação da incidência de cárie em pacientes com síndrome de Down após sua inserção em um programa preventivo
}

\author{
Evaluation of the incidence of dental caries in patients \\ with Down syndrome after their insertion in a preventive program
}

Aline Rogéria Freire deCastilho ${ }^{1}$

Sara Nader M arta ${ }^{2}$

${ }^{1}$ Departamento de O dontologia Infantil, Área deOdontopediatria, Faculdade de Odontologia dePiracicaba, Universidade Estadual deCampinas. Av. Limeira 901, CP 52. 13414-903 Piracicaba SP acastilho@fop.unicamp.br ${ }^{2}$ Universidade Sagrado Coração deJesus.
Abstract The objective of this work was to verify the incidence of dental caries by means of the CPO-D, CPO-S, ceo- $d$ and ceo-s indexes in patients with $D$ own syndrome regularly enrolled in a preventive program. Twenty four Down syndrome patients of both sexes age range of oneto 48 years were examined. The prevalence (initial experimental situation) and incidence (final experimental situation) of dental caries were verified using of theinitial and final CPO-D, CPO-S, ceo$\mathrm{d}$ and ceo-s indexes of the participants. From 24 individual examined, 10 (42.0\%) were free of caries. The prevalence of dental caries showed values of $C P O-D=2.33$; $C P O-S=3.60$; ceo- $d=$ 1.75 e ceo- $s=2.80$; while the incidence of caries showed values of $2.33 ; 3.80 ; 1.10$ e 1.90 , respectively. Down syndrome individuals evaluated in this study presented low level of caries and small incidence of new lesions, emphasizing the importance of the maintenance of these patients at preventive programs.

Key words Dental caries, Down syndrome, Preventive program
Resumo 0 objetivo deste trabalho foi verificar a incidência de cárie dentária por meio dosíndices CPO-D, CPO-S, ceo-d e ceo-sem indivíduos com síndrome de D own regularmentematriculadosem um programa preventivo. Foram examinados 24 indivíduos com síndrome de Down de ambos os sexos, com idade entre um e 48 anos. A prevalência ( situação experimental inicial) e a incidência (situação experimental final) de cárie dentária foram verificadas utilizando-se os índices CPO$D, C P O-S$, ceo-d e ceo-s iniciais e finais dos participantes. Dos 24 indivíduos examinados, dez $(42,0 \%)$ eram livres de cárie. A prevalência de cárie dentária mostrou valores de $C P O-D=2,33$; $C P O-S=3,60$; ceo- $d=1,75$ eceo- $s=2,80$; enquanto a inci dência decárieapresentou valores de2,33; 3,80; 1,10 e 1,90, respectivamente. O s indivíduos com síndrome de Down avaliados neste estudo apresentaram baixos índices de cárie e pequena incidência de novas lesões, enfatizando a importância da manutenção desses pacientes em programas preventivos.

Palavras-chave Cárie dentária, Síndrome de Down, Programa preventivo 
Introdução

Indivíduos com síndrome de Down apresentam, demodo geral, piores condições de higiene bucal; possivelmente, devido ao fato de que eles próprios realizam a sua higiene, e muitas vezes associasea essefato uma grandepermissividadepor parte dos pais em razão da presença da síndrome'.

O acesso aos cuidados de saúde bucal pelos pacientes com necessidades especiais é limitado, seja pela incapacidade dos cuidadores de identificar a causa ou avaliar adequadamente a condição bucal do paciente, seja pela incapacidade de les em expressar seu desconforto ou dor, ou ainda pela incapacidade de receber atendimento por alguns cirurgiões-dentistas que não se julgam aptos a realizar o tratamento odontológico nesses indivíduos? ${ }^{2}$.

Os programas de promoção de saúde bucal são ferramentas coadjuvantes no controle da cárie dentária, pois promovem um impacto positivo no controle da doença e, consequentemente, reduzem os valores dos índices de cárie ${ }^{3,4}$. 0 estudo realizado por Araújo $0^{5}$ mostrou que as crianças com síndrome de Down são suscetíveis à cárie dentária como qualquer outra criança e enfatizou a necessidade de acompanhamento odontológico desde o primeiro ano de vida. Neste estudo, $85 \%$ das crianças recebiam atenção odontológica e 15\% não recebiam, sendo que o maior número de dentes cariados foi observado nesteúltimo grupo.

Sabe-se hoje que o esforço do paciente, a motivação do dentista e um programa preventivo de saúde bucal bem planejado são fatores re levantes para o sucesso da prevenção de doenças bucais em indivíduos com síndrome de Down'.

A carência de dados com relação à incidência de novas lesões cariosas, assim como a efetividade desses programas e, especialmente, de programas direcionados aos indivíduos com síndrome de Down que, comprovadamente, apresentam piores condições de higiene bucal e necessitam de auxílio para a promoção de saúde bucal, são fatores indispensáveis para a verificação da qualidade dos serviços oferecidos einstigam essa investigação.

Assim, em razão das piores condições de higienebucal dessesindivíduose, consequentemente, sua maior vulnerabilidade às patologias bucais, este estudo tem como objetivo avaliar o impacto de um programa preventivo na incidência de cárie dentária em indivíduos com síndromede Down.

\section{M aterial e métodos}

\section{Grupo experimental}

A amostra foi composta por 24 indivíduos com síndrome de Down, deambos os sexos, com idadeentreum e 48 anos, regularmentematriculados no Programa de Assistência Integral ao Paciente Especial (PAIPE), sem distinção de raça ou cor, independentemente dos hábitos de higiene bucal ou alimentação. Destes indivíduos, dezoito eram crianças e seis eram adultos.

Esta amostra corresponde a $72,0 \%$ dos indivíduos com síndrome de D own participantes do PAIPE. Cinco indivíduos foram excluídos do estudo, poistiveram três faltas consecutivase, portanto, não participaram efetivamente do programa de prevenção. 0 restantefoi excluído porque apresentava deficiência mental severa, impossibilitando a realização do exame clínico, ou porque seus responsáveis não devolveram o termo de consentimento assinado. Os indivíduos com aparel ho ortodôntico fixo ou móvel eaquel es que fizeram uso de antibiótico sistêmico ou outros medicamentos que pudessem interferir nos resultados, mascarando-os, também foram excluídos dessa amostra. Esses dados foram obtidos por meio de uma entrevista prévia real izada com os responsáveis pelos pacientes.

Este projeto foi submetido ao ComitêdeÉtica e Pesquisa da Universidade Sagrado Coração de Jesus (processo no 046/06) e somente após sua aprovação e autorização dos pais ou responsáveis pelos pacientes iniciou-se a pesquisa.

Determinação da prevalência de cárie

Previamente aos exames clínicos intrabucais, foram feitos o treinamento e a calibragem do profissional no mês anterior ao do exame, de acordo com a Organização Mundial da Saúde (OMS), sendo examinados pelo menos 13\% da amostra, duas vezes, em dias consecutivos ${ }^{7}$, para que a calibração intraexaminador pudesse ser comprovada pela aplicação do teste Kappa, que éconsiderado como concordância"ótima" quando está no intervalo de 0,81-0,998.

Os exames clínicos foram realizados em 24 indivíduos por um único examinador previamente calibrado $(k=0,92)$, duas vezes. 0 objetivo do exame clínico foi obter os índices de cárie CPOD, CPO-S, ceo-d e ceo-s ${ }^{9}$, utilizando-se sonda exploradora n 5 com ponta romba para remoção de debris e espelho bucal plano, sob luz arti- 
ficial e após secagem prévia do elemento dentário com jato de ar.

0 índice CPO-D medeo ataque de cáriedental à dentição permanente. Suas iniciais representam respectivamente: dentes cariados (C), perdidos $(P)$, obturados $(O)$ e a medida de unidade que é o dente (D). Os perdidos subdividem-seem extraídos (E) e extração indicada (Ei). 0 índice ceo-d é correspondente ao CPO-D em relação à dentição temporária e inclui somente os dentes cariados (c), com extração indicada (e) e obturados (0). Exclui os extraídos, levando-se em conta as dificuldades para se identificarem os dentes perdidos devido à cárie ou pelo processo natural de esfoliação dentária?.

O CPO-D e o ceo-d médios para um determinado grupo foram obtidos pela divisão de todos os dentes atacados pelo número de indivíduos examinados. Os índices de cárie CPO-S e ceo-s são uma alternativa mais refinada para a aval iação do acometimento de cárie, cuja unidade demedida éa superfíciedental (s), eoscritérios de avaliação foram similares aos do índice CPO-D e ceo-d.

N enhuma recomendação quanto à dieta e higienização foi dada previamente ao exameinicial, e as condições dentárias foram anotadas em fichas individuais. Não foi realizado exameradiográfico complementar.

Posteriormente, os pacientes foram subme tidos à rotina de prevenção estabelecida no PAI$P E$, constituída por profilaxias profissionais mensalmente, orientações de higiene bucal (técnicas deescovação euso de fio dental) ao paciente e aos seus responsáveis.

\section{Determinação da incidência de cárie}

Decorridos 12 meses do exame clínico inicial, nova avaliação dos índices de cárie foi realizada, como já descrito no item anterior, com o objetivo de se verificar a incidência de novas lesões cariosas. Os dados anotados nas fichas apropriadas permitiram a comparação das condições inicial efinal.

\section{Análise dos resultados}

Os dados obtidos através do exame clínico bucal foram tabelados no programa Windows Excel para fins de comparação das situações inicial efinal.

\section{Resultados}

Valores baixos dosíndices de cárieCPO-D,CPO$\mathrm{S}$, ceo-d e ceo-s foram observados e uma alta porcentagem de indivíduos livres de cárie. Dos 24 indivíduos examinados, dez (42,0\%) eram livres de cárie, sendo nove crianças e um adulto.

Os valores médios dos índices de cárie CPO$D, C P O-S$, ceo-d eceo-siniciais efinais do grupo experimental estão apresentados na Tabela 1.

A prevalência de cárie dentária foi verificada por meio dos índices CPO-D, CPO-S, ceo-d e ceo-s, que tiveram valores médios de 2,33, 3,60, 1,75 e 2,80, respectivamente, representados pela situação experimental inicial.

Para verificar a incidência de cárie, utilizaramse os mesmos índices; entretanto, os valores encontrados foram 2,33, 3,80, 1,10 e 1,90, respectivamente, representados pela situação experimental final.

Os resultados mostraram a incidência de quatro novas lesões de cárie, demonstrada apenas pelo índice CPO-S, no período de avaliação. $\mathrm{N}$ ão houveincidência de cárienos demaisíndices utilizados.

\section{Discussão}

Sabe-se que a cárie dentária éuma doença infecciosa multifatorial e que muitos fatores podem influenciar potencializando ou amenizando a sua ocorrência - sendo a presença do biofilmebacteriano imprescindível para a ocorrência desta doença ${ }^{10}$. Assim, a implantação de programas preventivos, com foco na educação em saúde bucal enaremoção mecânica dessebiofilme, torna-se um importante aliado no controle desse agravo, principalmente em populações, como a enfocada neste estudo, de indivíduos portadores de síndrome de Down, em que as condições clínicas locais e sistêmicas podem ser dificultadoras desse controle.

Tabela 1. Valores médios dos índices CPO-D, CPO-S, ceo-d e ceo-s da população estudada.

\begin{tabular}{lcc}
\hline & Inicial & Final \\
\hline CPO-D & 2,33 & 2,33 \\
CPO-S & 3,60 & 3,80 \\
ceo-d & 1,75 & 1,10 \\
ceo-s & 2,80 & 1,90 \\
\hline
\end{tabular}


A avaliação das condições bucais em portadores de síndrome de D own, com relação à prevalência de cárie, doença periodontal, entre outros agravos, e sua relação com a própria patologia ou com as condições locais/ambientais tem sido objeto de numerosos estudos 1,3-6,11-17. Entretanto, poucos abordam a incidência dessa doença na síndrome de Down.

Fracasso et al. ${ }^{3}$ relacionaram a presença de cárie e a eficácia de um programa de promoção de saúde bucal nesses pacientes. Estes autores verificaram que o índice de cárie foi menor, além de haver maior porcentagem de crianças livres de cárie. Isto também foi aqui constatado: a incidência de cárie nos indivíduos com síndrome de Down participantes do PAIPE foi baixa equasea metade deles estavam livres de cárie, no período experimental aqui proposto, com apenas quatro lesões cariosas novas, na dentição permanente, perceptível apenas pelo índice CPO-S.

Os índices CPO-S e ceo-s, para superfícies cariadas, são utilizados como uma alternativa mais refinada para a avaliação do acometimento de cárie ${ }^{9}$. Os valores aqui encontrados parecem ser compatíveis com a realidade, uma vez que verificamos a ocorrência de cárie por superfíciee não apenas por dente afetado. Contudo, esses índices não puderam ser comparados com outros estudos, pois nenhum delesutilizou essecritério de avaliação.

Sabe-se que a idade dos indivíduos pode influenciar nos valores dos índices de cárie devido ao fator cumulativo desses índices ${ }^{18}$ ou ao maior tempo de exposição dos dentes na cavidade bucal. No levantamento epidemiológico da $\mathrm{OM} \mathrm{S}^{9}$, a prevalência decárieaumentou em razão da idade dos participantes. Em nosso estudo, seis indivíduos eram maiores de 12 anos, e destes dois apresentaram novas lesões decárie, refletidos pelo aumento no CPO-S, de 3,60 para 3,80. Desta forma, a idade dos participantes pode explicar a incidência, mesmo que pequena, de cárie observada neste estudo.

A carência de dados na literatura de estudos que abordam a incidência de cárie em um determinado período, com o objetivo de medir o impacto deuma abordagem preventiva, como o que foi aqui proposto, dificulta a comparação com os resultadosaqui obtidos, porém M oura et al. ${ }^{4}$ também verificaram a presença de cárie dentária em crianças que frequentavam um programa preventivo. Os autores observaram que o programa teve um impacto positivo no controle da doença cárie, pois as crianças apresentaram baixo CPO$D$, apesar da presença de novas lesões.
Esse impacto positivo, com mudanças significativas do padrão de higiene bucal dos participantes deste estudo, bem como de seus responsáveis na importância dada às questões de saúde bucal, deve ser evidenciado. Embora com dificuldades para o comparecimento frequente nas consultas agendadas, a incidência de novas lesões cariosas nesses indivíduos foi baixa, após 0 início do acompanhamento. Esse fato deve ser entendido como alerta para que se destine mais atenção a determinados indivíduos, necessitando em muitas vezes a diminuição no intervalo entre as consultas e a busca de possíveis causas que possam estar propiciando 0 aparecimento de novas lesões, significando que a doença cárie não está controlada.

Devido à extensa faixa etária dos pacientes que compuseram esta amostra, optou-se pela avaliação de acordo com a presença das dentições decídua e permanente, e não apenas pela idade cronológica dosindivíduos. Assim, o valor do CPO-D de 2,33, menor que o do ceo-d de 3,60 , aqui encontrado, difere dos resultados encontrados por Moraes et al..$^{12}$, com CPO-D de 2,68 e ceo-d de 1,15, em que os indivíduos que participaram do estudo não recebiam atendimento odontológico na ocasião do exameclínico ou foram divididos por faixa etária (3-9, 10-17, 18-28 anos), o que, provavelmente, interferiu nos valores dos índices de cárie analisados.

A relação entre o índice de cárie e a condição do paciente em ser institucionalizado ou não foi avaliada por Giro et al. ${ }^{15}$, os quais verificaram que indivíduos com necessidades especiais institucionalizados ou não apresentaram valores altos deCPO-D (8,39 e 5,96, respectivamente). Dos institucionalizados, apenas 7,7\% eram livres de cárie e 34,7\% dos não institucionalizados estavam nesta condição. A população avaliada em nosso estudo não era institucionalizada, porém a porcentagem deindivíduoslivres decáriefoi muito maior do que aquela do estudo de Giro et al. ${ }^{15}$. Esses valores se devem, provavelmente, à participação desses indivíduos no programa preventivo-educativo em questão, com envolvimento efetivo de seus responsáveis, evidenciando a importância da cumplicidade entre paciente-profissional-família em busca de um objetivo comum.

A intensificação das orientações de educação e higiene bucal aos pacientes e/ou responsáveis pelos profissionais do PAIPE tem sido uma prática rotineira, com envolvimento dos responsáveis, tornando-os parceiros e com o propósito de fazer com que o trabalho de prevenção da cárie dentária se estenda ao ambiente domiciliar 
enão fique restrito apenas às visitas ao consultório odontológico. N esse sentido, evidencia-se a importância da relação harmoniosa profissional-família, sendo queo envolvimento efetivo dos cuidadores desses pacientes com as rotinas do programa é fundamental para a manutenção da sua saúde bucal.

A literatura apresentada evidencia a necessidade e a importância dos programas preventivos para a promoção e a manutenção da saúde bucal da população e demonstra a escassez não apenas dos referidos programas, mas também de estudos direcionados aos pacientes com necessidades especiais.

\section{Conclusão}

Osindivíduos com síndrome de D own avaliados neste estudo apresentaram índices de cárie baixos com incidência de novas lesões praticamente desprezíveis no período avaliado. Os resultados encontrados enfatizam a importância da implantação de programas preventivos contínuos para controle da cárie dentária, direcionados não apenas aos indivíduos com síndrome de D own, mas também àqueles com outras necessidades especiais, a fim de se promover a saúde bucal e a qualidade de vida dessa população.

\section{Referências}

1. Pinazo JDC, Vianna MIP, Lopes FL. Cárie dentária e a placa bacteriana em crianças de 7 a 14 anos portadoras da síndrome de Down, matriculadas em instituições públicas e privadas do município de Salvador-Bahia. Rev Fac Odont UFBA 1998; 17:15-24.

2. Hennequim M, Faulks D, Roux D. Accuracy of estimation of dental treatment need in special care patients. J Dent 2000; 28:131-136.

3. Fracasso M LC, Rios D, Provenzano M GA, Goya $S$. Efficacy of an oral healh promotion program for infants in the public sector. J Appl Oral Sci 2005; 13(4):372-376.

4. Moura LFAD, Moura MS, Toledo OA. Dental caries in children that participated in a dental program providing mother and child care. J Appl Oral Sci 2006; 14(1):53-60.

5. Araújo NCBI. Prevalência de cárie dentária em crianças portadoras de Síndrome de Down na faixa etária de 0 a 60 meses. J Bras 0 dontopediatr 0 dontol Bebe 2000; 3(12):147-157.

6. Shapira J, Stabholz A. A comprehensive 30-month preventive dental health program in a pre-adolescent population with Down's syndrome: a longitudinal study. Spec Care Dentist 1996; 16(1):33-37.

7. Organização M undial da Saúde. Levantamentos básicos em saúde bucal. $4^{a}$ ed. São Paulo: Santos; 1999.

8. Pereira MG. Epidemiologia: teoria e prática. Rio de Janeiro: Guanabara Koogan; 1999.

9. Organização Mundial da Saúde. Fichas de avaliação. In: World Health Organization. Oral health surveys basic methods. $4^{\text {th }}$ ed. Geneva: WH O; 1997. p. $21-50$

10. Thylstrup A, Fejerskov O. Cariologia clínica. $3^{\mathrm{a}} \mathrm{ed}$. São Paulo: Santos; 2001.

11. Ulseth JO, Hestnes A, Stovner LJ, Storhaug K. Dental caries and periodontitis in persons with Down syndrome. Spec Care Dentist 1991; 11(2):71-73.
12. M oraes MEL, Bastos MS, M oraes LC, Rocha JC. Prevalência de cárie pelo índice CPO-D em portadores de síndrome de Down. Pós-Grad Rev Odontol 2002; 5(2):64-73.

13. Vazquez CR, Garcillan MR, Rioboo R, Bratos E. Prevalence of dental caries in an adult population with mental disabilities in Spain. Spec Care Dentist 2002; 22(2):65-69.

14. Bradley C, McAlister T. The oral health of children with Down syndrome in Ireland. Spec Care Dentist 2004; 24(2):55-60.

15. Giro EM A, Orrico SRP, Campos JADB, Lorena SM, Cortez LMS. Caries prevalence in institucionalized and non-institucionalized disable patients: consumption of simple carbohydrates. Rev 0 dontol Unesp 2004; 33(2):75-79.

16. Lee SR, Kwon HK, Song KB, Choi YH. Dental caries and salivary immunoglobulin $\mathrm{A}$ in Down syndrome children. J Paediatric Child H ealth 2004; 40:530-533.

17. Fung K, Allison P. A comparison of caries rates in non-institutionalized individuals with and without Down syndrome. Spec Care Dentist 2005; 25(6):302310.

18. Brasil. Ministério da Saúde. Secretaria de Atenção à Saúde. Departamento de Atenção Básica. Coordenação Nacional de Saúde Bucal. Levantamento epidemiológico em Saúde Bucal: 1ae etapa - cárie dental - projeto. Brasília: Ministério da Saúde; 1996.

Artigo apresentado em 23/08/2007

Aprovado em 12/11/2008

Versão final apresentada em 13/12/2007 